\title{
Towards a Ubiquitous Child Emergency App - Ideas to Simplify and Ensure the Machine-2-Machine Communication
}

\author{
Michael SCHMUCKER ${ }^{\mathrm{a}, 1}$ and Martin $\mathrm{HAAG}^{\mathrm{a}}$ \\ ${ }^{a}$ Heilbronn University of Applied Sciences, GECKO Institute
}

\begin{abstract}
Medical emergencies involving children are rare events. The experience of emergency physicians is therefore low and the results are correspondingly poor. Assistance services to help in emergencies are regularly requested. The use case is thus very complicated, a complex system consisting of multiple devices is necessary to provide the most efficient and effective service. This short paper presents prototypically tested ideas on how such a ubiquitous approach can be designed and how communication between devices can be simplified and ensured.
\end{abstract}

Keywords. mHealth, uHealth, Wearable Devices, Paediatrics, Emergencies

\section{Introduction}

Pediatric resuscitation outcomes are poor, too few children survive with favorable neurological outcome [1]. There are many reasons for this, e.g. lack of routine and training [2], in addition emergency physicians are particularly stressed in such cases [3]. Therefore, new developments are needed. Technical aids are repeatedly desired [4], but they are difficult to integrate into the process. Various devices are now available, e.g. smartphones and several wearables. Each device itself has strengths but also weaknesses that make it difficult to use. A smartphone, e.g. can be used to detect the size of a child $[5,6]$, from which a variety of values can be derived. A smartwatch can measure the compression depth during cardiac massage [7]. A Head-Mounted-Display (HMD) is suitable for displaying information according to the principle of Calm Technology [8], but the control of an HMD is complicated. Therefore, it needs a complex system of various devices that need to communicate with each other. In this paper, prototypically tested ideas are presented that can help to develop such a system.

\section{Methods}

Based on previous research, as described in [5] and [9] an assistance service was designed that best meets the requirements for efficiency and effectiveness. Subsequently, the application was prototypically developed using state-of-the-art protocol development techniques [10] and its feasibility was tested in an internal non-representative study.

\footnotetext{
${ }^{1}$ Corresponding Author: Michael Schmucker, Heilbronn University of Applied Sciences, Max-Planck-
} Str. 39, 74081 Heilbronn, Germany; E-mail: michael.schmucker@hs-heilbronn.de. 


\section{Results}

The goal was to create an application that was as simple as possible for the user and secure in terms of communication. Communication is initiated via a QR code on the smartwatch, which is scanned by the HMD and smartphone and contains all necessary information. Further, it is important to send as little information as possible, as it is less error-prone and preserves the battery. For example, saving the IP in Hex can save up to $33 \%$ ( 8 instead of 12 characters). To prevent the communication channel from collapsing, it is redundant (Bluetooth and WiFi). To transmit the information, a protocol was developed that contains 3 pieces of information (type, event, value) and is sent using Android RemoteIntents. These had a transmission speed of 4-40ms in an internal, nonrepresentative study. Error handling was implemented using a positive acknowledgement method. At-least-once was taken as the error semantics.

\section{Discussion}

According to our research, the best possible assistance service is based on a ubiquitous approach, more precisely the interaction of smartphone, HMD and smartwatch. With the smartphone, the size can be measured $[6,7]$, the computing power is sufficient to provide the server. However, it can be poorly operated and seen. The glasses can be used very well for displaying information, but the control is not intuitive. The smartwatch can significantly improve the quality of treatment with its sensors [8]. Typical for smartwatches is also a difficile tactile feedback with different vibration patterns on the wrist, whereby feedback can be transmitted to the treating physician not only via the HMD. The application can also be controlled via the smartwatch. A successful, secure and as simple as possible connection could be established at least under laboratory conditions. A test under real conditions in a simulation center is still pending. However, the ideas and results developed can still provide ideas and help other researchers. In conclusion, assistance services for this complex process is possible, but a lot of research is still needed in the areas of automation and usability. Advances in technology, such as depth cameras and better processors in HMDs, can accelerate this process.

\section{References}

[1] Sutton RM et al. Low-Dose, High-Frequency CPR Training Improves Skill Retention of In-Hospital Pediatric Providers, Pediatrics 2011;128:145-151.

[2] Die Angst fährt mit zum Einsatz, Available at: https:/www.aerztezeitung.de/medizin/krankheiten/ herzkreislauf/article/858981/kinder-notfaelle-angst-faehrt-einsatz.html, Accessed January 15, 2021.

[3] Zink W et al. Invasive Techniken in der Notfallmedizin, Der Anaesthesist 2004;53:1086-1092.

[4] Kaufmann J et al. Medikamentenfehler bei Kindernotfällen - eine systematische Analyse, Dtsch Arztebl Int. 2012;109:609-616.

[5] Schmucker M et al. Evaluation of Depth Cameras for Use as an Augmented Reality Emergency Ruler, Stud Health Technol Inform 2019;260:17-24.

[6] Wetzel $\mathrm{O}$ et al. A smartphone application to determine body length for body weight estimation in children: a prospective clinical trial, J Clan Monat Comput 2018;32:571-578.

[7] Gruenerbl A et al. Smart-watch life saver: smart-watch interactive-feedback system for improving bystander CPR, Proceedings of the 2015 ACM International Symposium on Wearable Computers.

[8] Weiser M, Brown JS. Coming Age of Calm Technology, Beyond Calculation, Springer, New York; 1997.

[9] Schmucker M et al. Speech Recognition Evaluation of a State-Of-The-Art Smartglass in the Use Case Pediatric Resuscitation, Stud Health Technol Inform. 2019;262:67-70.

[10] H. König, Protocol Engineering, Springer-Verlag, Berlin Heidelberg; 2012. 